\title{
ANALISIS DE LA RESISTENCIA ESPECÍFICA DE LOS JUGADORES DE LA SELECCION DE FUTBOL DE COSTA RICA A TRAVES DEL TEST DE PROBST
}

\author{
LOZANO R. ${ }^{1}$, BARAJAS, ${ }^{\text {Y. }}{ }^{1}$ \\ Docente Universidad de Pamplona, Programa de Educación Física
}

\section{RESUMEN}

El propósito de este estudio fue identificar el Umbral Anaeróbico y la cualidad aeróbica, en los jugadores de la Selección de fútbol de Costa Rica, quienes participaran en la Copa del Mundo FIFA, Brasil 2014. El protocolo llevado a cabo, en las instalaciones de la Federación de Fútbol de Costa Rica, fue el test de Probst (1989), utilizando el software TIVRE Fútbol, siendo un test de campo reconocido a nivel internacional, para determinar la resistencia específica, del jugador en su propio terreno de entrenamiento y competición, se evaluaron 23 jugadores profesionales, de género masculino. El protocolo desarrollado por Probst, consiste en recorrer espacios señalados a velocidades especificas las cuales se incrementan en cada periodo, cada sujeto testeado utilizó un medidor de frecuencia cardiaca marca Polar, para el control de la frecuencia cardiaca, que posteriormente fue importante para la determinación del umbral anaeróbico, utilizando la metodología de Conconi y cols $(1982,1996)$, a través de la deflexión de la curva de frecuencia

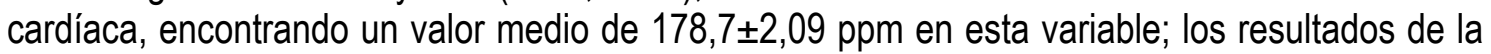
velocidad máxima promedio fueron de $15,22 \pm 7,90 \mathrm{~km} / \mathrm{h}$; estando en el período número $18,27 \pm 5,96$ del test, con esta velocidad y en este período, se lograron distancias promedio de $4101,67 \pm 87,69$ metros. Dentro de estos resultados se estimó el consumo máximo de oxígeno encontrando un valor de $54,23 \pm 3,72 \mathrm{ml} \cdot \mathrm{kg}^{-1} \cdot \mathrm{min}^{-1}$, determinado indirectamente mediante la fórmula propuesta por el Colegio Americano de Medicina del Deporte (1999).

Palabras clave: Test de campo interválico (TIVRE-Fútbol), Umbral anaeróbico. Deflexión de la frecuencia cardiaca. Control de la frecuencia cardiaca. Jugadores de fútbol.

\section{ANALYSIS OF SPECIFIC RESISTANCE OF PLAYERS SELECTION OF COSTA RICA SOCCER ACROSS THE TEST OF PROBST}

\begin{abstract}
The purpose of this study was to identify the anaerobic threshold and aerobic qualities in players football team Costa Rica, who participate in the FIFA World Cup Brazil 2014 . Protocol held at the premises of the Football Federation of Costa Rica, was the test of Probst (1989), using the TIVRE Football software, being a field test recognized internationally, to determine the specific resistance of the player in his own field of training and competition, is evaluated 23 professional players, male. Developed by Probst, protocol is to travel to specific fields marked speeds which increase in each period, each subject tested used a Polar heart rate meter mark, to control heart rate, which was subsequently important for determining the anaerobic threshold, using the methodology of Conconi et al $(1982,1996)$ through the deflection curve of heart rate , by finding an average value of $178.7 \pm 2.09 \mathrm{ppm}$ in this variable; results were average top speed of $15.22 \pm$ $7.90 \mathrm{~km} / \mathrm{h}$; number being in the period $18.27 \pm 5.96$ test, with this speed and in this period, average distances of $4101.67 \pm 87.69$ meters was achieved. Within these results the maximum oxygen consumption was estimated and found to be $54.23 \pm 3.72 \mathrm{ml} . \mathrm{kg}-1 . \mathrm{min}-1$, indirectly determined by given by the American College of Sports Medicine (1999) formula .
\end{abstract}


Keywords: Test Interval field (TIVRE-Football), anaerobic threshold. Deflection of the heart rate. Control of the heart rate. Soccer players.

\section{INTRODUCCION}

En las diferentes disciplinas deportivas, tanto cíclicas como las acíclicas, se hace necesario conocer su máxima condición física, esto controlado a través de diferentes test tanto de campo o laboratorio (Lozano y cols., 2011), de esta manera determinar parámetros tan importantes como lo es el $\mathrm{VO}_{2}$ max., el umbral anaeróbico (UA), siendo factores determinantes del rendimiento deportivo. (López y Legido, 1991). Y logrando optimizar la preparación de los deportistas a través de la correcta planificación, teniendo en cuenta el seguimiento realizado a través de la temporada.

En las disciplinas de equipo o conjunto, sus valores tanto de $\mathrm{VO}_{2}$ máx., como de UA, son elevados en relación a personas sedentarias en general (García y Cols, 2003 ; Bangsbo, 1996; 1998), pero no son tan elevados como las disciplinas de resistencia, alcanzando' valores superiores a los 65 ml.kg.min. (Franco, 1998; Bosco, 1991).

También es de vital importancia realizar el control de la frecuencia cardiaca (FC) y la velocidad de carrera, con la finalidad de optimizar el rendimiento de estos deportistas (Lozano, 2009, Lozano y cols, 2011). La realización de estas pruebas se llevan a cabo principalmente en el laboratorio, donde estos resultados son más exactos y precisos, pero con el inconveniente que no son específicos al deporte, al igual que requieren de un mayor costo, por los equipos utilizados allí; de esta forma la realización de test de campo nos ofrecen resultados más reales al deporte, es decir más específicos (Lozano y cols, 2011), siendo necesario en la realización de estos test, una cantidad menor de equipos y de menor costo, al igual un fácil análisis de los resultados. Para el control de estos test en el campo es fundamental contar con software informáticos, los cuales nos guían en el transcurso de las evaluaciones (García y cols, 2003).

El UA es la zona de intensidad en la cual el sistema de energía aeróbico no puede por sí solo suplir las demandas energéticas del sujeto (López Chicharro, J., 2004) por lo tanto se acude al metabolismo anaeróbico, el cual es un factor importante para el alto rendimiento deportivo (López y Legido,1991) ; utilizando el método de Conconi y cols (1982, 1996), lo podemos determinar a través de una serie sucesiva de esfuerzos que se van incrementando en intensidad, al realizar el seguimiento de la FC, durante estos esfuerzos encontramos que los primeros estadios o períodos la FC se comporta de forma lineal, teniendo en cuenta la intensidad del esfuerzo, la cual es totalmente proporcional, hasta alcanzar una deflexión de la curva hacia la derecha, lo cual corresponde al UA, a partir de este punto se pierde la proporcionalidad y no se alcanzan aumentos exagerados, en cuanto a la FC, de acuerdo al protocolo seleccionado.(Villa J. y cols, 2000, 2007).

De esta manera, la literatura nos muestra, que el test más apropiado para controlar las variables fisiológicas, susceptibles a los cambios generados por el entrenamiento y específicamente para el fútbol, es el test de Probst (1989), con el cual podemos determinar la resistencia específica del jugador en su propio terreno de entrenamiento y competición, siendo un test de campo óptimo para su aplicación y fácil interpretación de los resultados, de esta manera desarrollar una mejor preparación deportiva, para la competición. (Lozano y cols, 2011)

\section{DESARROLLO METODOLOGICO}

Se determinó el Umbral Anaeróbico y la capacidad aeróbica, con los jugadores de la Selección Nacional de Fútbol de Costa Rica, durante un microciclo de preparación, para participar en la Copa del Mundo FIFA 2014, Brasil, durante el mes de diciembre del año 2013, en las instalaciones de la Federación de este país, para ser utilizado 
posteriormente en la dosificación de la carga correspondiente al desarrollo de la resistencia específica y lograr el máximo rendimiento, aplicable a esta disciplina deportiva.

Participaron en el presente estudio 23 sujetos, todos profesionales, pertenecientes a diferentes ligas del mundo y de la propia Federación Costarricense, todos de sexo masculino; la valoración se realizó con previa autorización por escrito por cada uno de ellos y de su entrenador Jorge Luis Pinto, quien luego de su grandiosa clasificación al torneo más importe a nivel internacional, ha propuesto desarrollar este estudio, preparatorio para la Copa del Mundo 2014 a llevarse a cabo en Brasil, en el mes de Junio y teniendo en cuenta los requerimientos internacionales, cuando se trabaja con seres humanos.

\section{Variables}

Las variables controladas y analizadas en el presente estudio fueron la distancia alcanzada durante el test, velocidad en el último período, frecuencia cardiaca al final de cada periodo, con estos datos se obtuvo el umbral anaeróbico tanto con su respectiva FC y velocidad de carrera, durante el test de campo Probst, controlado a través del software TIVRE-Fútbol versión 2.0 y al finalizar se determinó el volumen máximo de oxigeno de forma indirecta, por medio de la ecuación recomendada por el ACSM (1999).

\section{Procedimiento}

Los deportistas de la Selección de Fútbol de Costa Rica, recibieron instrucciones del protocolo a seguir, que correspondió a un circuito marcado con 14 puntos, cada punto señalado con un cono y ubicado a una distancia de 10 metros entre cada uno; el punto de partida era el mismo de finalización de cada uno de los periodos, el recorrido marcado está diseñado con diversos cambios de dirección, que se asemejan a los realizados en este deporte, el futbolista se desplazó a cada marca de referencia guiado por una señal auditiva (bips), en cada cono correspondía a un bip, el test se inició a una velocidad de $6 \mathrm{~km} / \mathrm{h}$, correspondiendo al período número 4 , el cual mantuvieron por dos vueltas completas al circuito (Figura 1), equivalentes a 280 metros, al cumplirse este recorrido se efectúo una pausa de 30 segundos, donde el deportista recuperaba libremente, sin alejarse del cono, faltando 3 segundos para completar el tiempo de recuperación se daba una cuenta regresiva para que el deportista estuviera en disposición de salir a realizar el siguiente período, que comprendió en un incremento en la velocidad de $0,6 \mathrm{~km} / \mathrm{h}$, hasta llegar al agotamiento 0 que el sujeto no alcanzara dos conos seguidos de acuerdo al bip o señal acústica; estos periodos se programaban en el software Tivre-Fútbol versión 2.0, el cual estaba instalado en un computador portátil y cuya señal era emitida por dos parlantes externos, lo suficientemente potentes para escucharse claramente en todo el campo; este protocolo se desarrolló atendiendo la metodología descrita por Probst (1989).

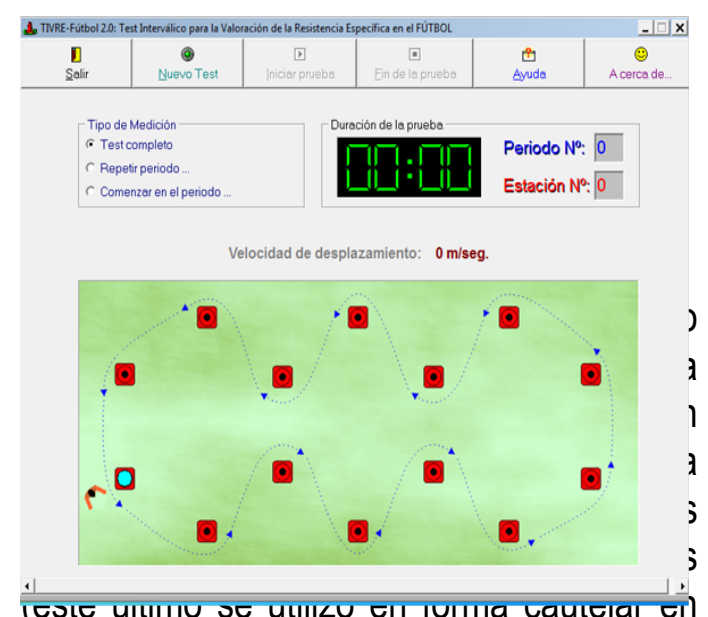
caso de un fallo tecnológico del equipo utilizado), este control de la FC fue importante para la determinación del UA, utilizando la metodología desarrollada por Conconi y cols $(1982,1996)$. Los periodos pares se realizaron en el sentido en que giran las manecillas del reloj y los impares en sentido contrario.

El presente estudio se desarrolló en la Sede de la Federación Costarricense, en su principal campo de fútbol de entrenamiento, 
a una altitud de 1160 metros sobre el nivel del mar y una temperatura promedio de 21 ${ }^{\circ} \mathrm{C}$ (Figura 2), las condiciones climáticas fueron favorables para poder realizar sin inconvenientes el test a todos los deportistas, su ubicación exactamente fue en la ciudad capital San José de Costa Rica. Donde esta selección ha sido una de las más laureadas en Centro América y va asistir a su 4 copa del mundo.

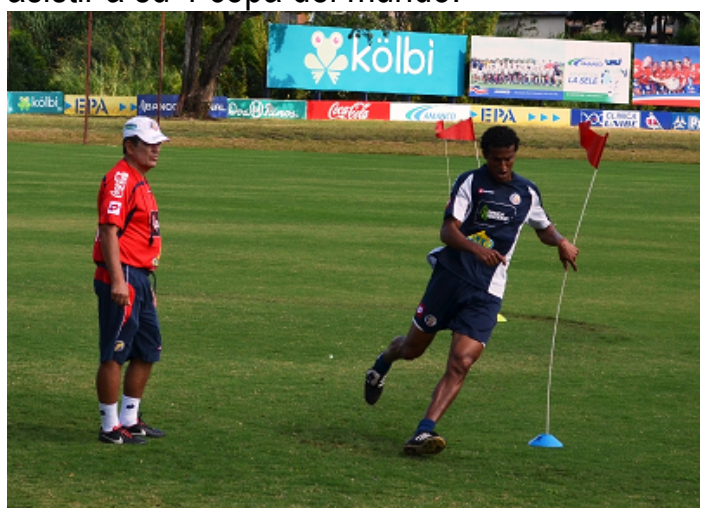

Figu9r5a 2. Desarrollo del test en sede Selección de Fútbol de Costa Rica

Se solicitó al Preparador Físico, que los sujetos a evaluar no realizaran ningún tipo de actividad física excesiva en el transcurso de 12 horas previas a la evaluación. De la misma forma, se recomendó que utilizaran la indumentaria habitual para práctica del fútbol.

Antes de dar inicio al test se estandarizó un calentamiento, durante 10 minutos realizado por el preparador físico del equipo, donde en cada bloque participaban 8 jugadores para tener un mejor control sobre cada uno de ellos. Una vez terminado el calentamiento cada acompañante de campo guiaba al jugador hacia un cono determinado y que se encontraba equidistante de los otros puntos de salida, para evitar que en el momento de la prueba pudieran existir interferencias durante el recorrido. Una vez ubicado los jugadores en su punto de referencia, el director de la prueba daba inicio al test. De la misma forma, en el desarrollo del test se motivó constantemente a los sujetos de forma verbal buscando el máximo rendimiento.

\section{Análisis de la información}

Los resultados de frecuencia cardiaca, fueron descargados de los pulsómetros Polar a través del software Polar Precisión versión 4.03.050 (Polar Electro Oy, 2007), donde se analizó sujeto por sujeto, el comportamiento de los puntos de FC más alta de cada uno de los periodos y el correspondiente más bajo en cada recuperación, estos datos se llevaron a una hoja de cálculo Excel 2007., donde se graficó, la cinética de la FC en el transcurso del test, para que de esta manera se comprobara la curva de deflexión de la FC, de acuerdo a la metodología realizada por Conconi y cols $(1982,1996)$.

Estos datos recibieron un tratamiento estadístico por medio del software SPSS v. 19., a través de una análisis descriptivo, donde los resultados se muestran con valores medios del grupo evaluado, con su correspondiente error estándar de la media (EEM), ubicando de esta manera el resultado de cada sujeto con el promedio del grupo, muy seguramente con el desarrollo de una segunda valoración y ver los cambios producidos por la preparación, podremos correlacionar y observar un análisis más detallado de estas variables determinadas en el presente estudio.

\section{RESULTADOS}

En la tabla 1, se observan los resultados de los 23 sujetos, que se preparan para participar en la Copa del Mundo Brasil 2014, representando a la Selección de Costa Rica, mostrándose como valores medios y su correspondiente error estándar de la media; encontrando que el resultado de la velocidad máxima fue de $15,22 \pm 7,90 \mathrm{~km} / \mathrm{h}$; que equivalió al período número $18,27 \pm 5,96$ del Test Interválico de Probst (1989), con esta velocidad y en este período, se lograron distancias medias de 4101,67士 87,69 metros y el tiempo total en que se

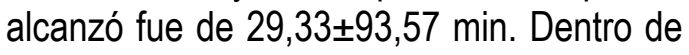
estos resultados se estimó el consumo máximo de oxígeno $\left(\mathrm{VO}_{2}\right.$ máx.) encontrando un valor medio del grupo de $54,23 \pm 3,72$ ml.kg-1. min-1, determinado indirectamente mediante la fórmula propuesta por el 
Colegio Americano de Medicina del Deporte (1999).

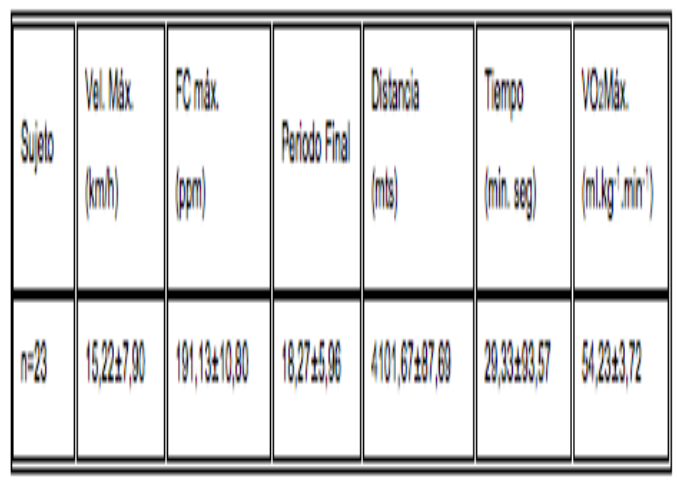

Tabla 1. Valores Máximos del Test de Campo Probst

En la figura 3, se observan los valores de velocidad máxima promedio de los sujetos evaluados, identificados con la barra azul, contrastados con la velocidad en el umbral determinada en el transcurso del test por medio de la deflexión de la FC, utilizando la metodología descrita por Conconi y cols $(1982,1996)$. En esta figura se encuentran diferencias significativas, mostrando que la velocidad en umbral fue alcanzada al 79,90 $\pm 3,57 \%$ del valor máximo obtenido en el test interválico de Probst, que corresponde a $15,22 \pm 7,90 \mathrm{~km} / \mathrm{h}$, es decir la velocidad umbral en promedio fue alcanzada a una velocidad de carrera de $12,16 \pm 0,85 \mathrm{~km} / \mathrm{h}$, correspondiendo entre el período 14 y 15 , del protocolo original, teniendo en cuenta el software, pero como los deportistas son profesionales, ellos iniciaron desde el periodo 4 , es decir que esta variable se determinó exactamente entre el período 11 y 12, al tener en cuenta este inicio y el promedio del máximo período fue el 18 es decir 3 por encima de este valor determinado en la velocidad de umbral anaeróbico.

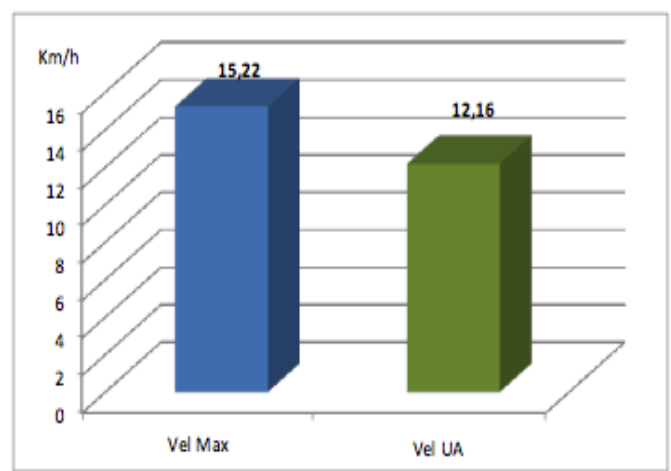

Figura 3. Valores de velocidad máxima y en umbral, del test de Probst.

Teniendo en cuenta la frecuencia cardiaca máxima obtenida durante el test interválico de Probst (1989), y utilizando la metodología descrita por Conconi y cols (1982, 1996), se determinó que el umbral anaeróbico promedio para el grupo de sujetos evaluados, fue alcanzada a una FC de 178,7 $\pm 2,09$ ppm, lo que corresponde al $93,47 \pm 2,09 \%$, teniendo en cuenta que la FC máxima determinada fue de $191,13 \pm 10,80$ ppm, y esto al momento se observar la deflexión de la cinética de la FC, determinado durante el protocolo establecido de campo, para conocer la resistencia específica de los Jugadores de la Selección Nacional de Fútbol de Costa Rica, encontrándose estos valores dentro de los rangos establecidos en la literatura (Volko y col, 1989; Barrios y col., 1995; Garcia, M. y cols 1996). Los valores se observan en la figura 4.

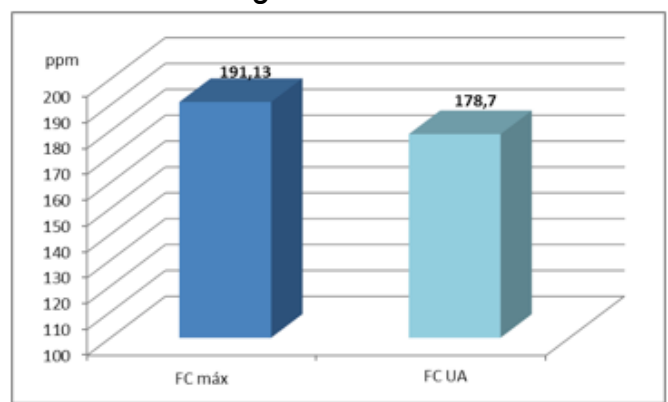

\section{¡Error! No se pueden crear objetos} modificando códigos de campo.

Figura 4. Valores de la Frecuencia Cardiaca máxima y en umbral anaeróbico

Al momento de observar la cinética de la frecuencia cardiaca, en el transcurso del test de campo, realizado a los jugadores profesionales de la Selección Nacional de Costa Rica, observamos que fue totalmente lineal y progresiva de acuerdo a la intensidad del esfuerzo y que aproximadamente en el período 14 al 15 se determinó este parámetro fundamental para adaptar las cargas de trabajo, esenciales para optimizar el rendimiento deportivo. $Y$ más aun que ellos se están preparando para participar en la Copa del Mundo, Brasil 2014. Estos datos se observan en la figura 5. 


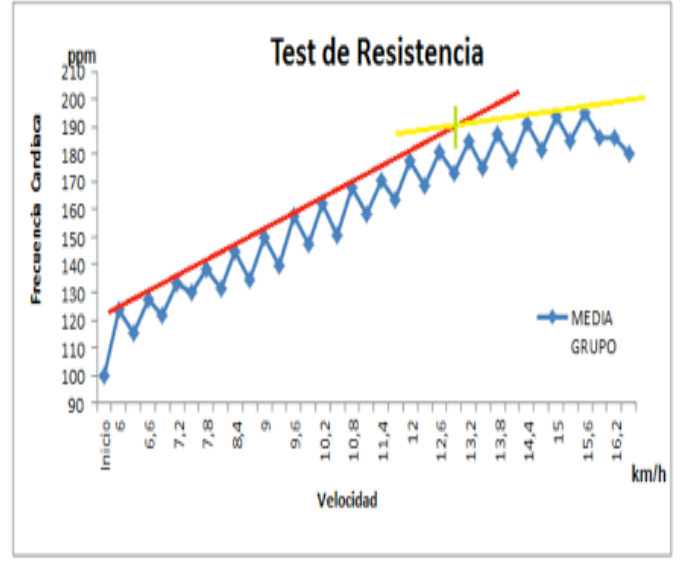

Figura 5. Cinética de la FC en el transcurso del test, promedio de todo el grupo.

\section{DISCUSION}

Uno de los aspectos más importantes a destacar en la presente investigación, ha sido la determinación del umbral anaeróbico, por medio de la deflexión de la frecuencia cardiaca, durante el test de Probst, esto determinado por medio de la metodología desarrollada por Conconi $F$. (1982, 1996), sin embargo es un parámetro muy criticado a nivel internacional, ya que algunos autores en la literatura manifiesta que es muy improbable detectar esta inflexión de la FC (Lopez-Calbet, 1995); no obstante otros autores manifiestan que es una metodología aceptable, de fácil uso, no invasiva y menos costosa, que otros protocolos, donde se observa con claridad este punto de inflexión de la $\mathrm{FC}$, determinando con facilitad el Umbral Anaeróbico, no solamente en futbolistas, jugadores de baloncesto y patinaje de carreras (Garcia, J. y cols, 2003; Lozano y cols, 2011; Villa J., y cols 2004; Hoftman y cols., 1994, 1997).

En este orden de ideas, observamos que la detección del umbral anaeróbico, por medio de la inflexión de la FC, realizándolo de una forma manual, es más fácil y reproducible a través de la temporada para realizar un seguimiento a los deportistas y poder tener un referentes y mejor análisis para desarrollar una adecuada planificación de las cargas externas y poder optimizar el rendimiento deportivo. (Conconi y cols, 1982, 1996; Lozano y cols, 2009 y 2011).
Es por estas razones y como lo ha establecido Conconi F. (1982, 1996), que los primeros estadíos o períodos, no hay un aumento significativo y progresivo de acuerdo a la intensidad del esfuerzo, de esta manera al momento de hallar el punto de inflexión, se deben omitir estos períodos y a partir de una intensidad más elevada, se obtendrá un aumento totalmente lineal $y$ progresivo de la cinética de la FC, para poder determinar con mayor precisión el umbral anaeróbico de una forma manual, hechos fundamentales para la determinación de esta importante variable y que a través de las diferentes publicaciones nos han mostrado con precisión la determinación de este parámetro. (Lozano y col, 2009 y Lozano y cols, 2011, Villa J., y cols, 2007; Garcia, J y cols, 2003)

De esta manera las disciplinas de resistencia (deportes cíclicos) el $\mathrm{VO}_{2}$ máx y en especial el UA, son determinantes en el rendimiento deportivo, siendo mayores a los observados en las disciplinas de equipo (deportes acíclicos). En cambio, en estos son mayores a los encontrados en sedentarios 0 en disciplinas de corta duración. (Reilly, T., 1997; Bangsbo, J., 1998)

López - Calbet y cols (1995) calcularon la reproducibilidad del Test de Conconi repitiendo un test sobre la banda $\sin$ fin 6 veces a lo largo de 3 meses en 23 estudiantes de educación física, concluyendo que la velocidad en el dicho umbral variaba en un $12-15 \%$, mientras que la frecuencia cardiaca sólo variaba un 5 $\%$. Sin embargo, y como demuestran estos mismos autores, mientras que la velocidad y la FC en el umbral pueden oscilar entre un 0 - $20 \%$, estas variables sólo oscilaron entre un $0-8 \%$ al final del ejercicio. En este sentido, el Test de Probst (1989), independientemente de su validez o no en la determinación del UA interválico, por medio de la metodología desarrollada por Conconi y cols (1982, 1996), aporta 
información valiosa por encima de los correspondientes al hipotético UA; lo que podría corresponderse con un mejor potencial o capacidad anaeróbica, logrando optimizar el rendimiento deportivo, a través de estas variables tan valiosas, controladas a lo largo de la temporada y de esta manera aplicar uno de los principios del entrenamiento deportivo con mayor eficacia, como lo es la especificidad e individualización de las cargas.

Sin embargo, en el presente estudio la relación entre la FC e intensidad de esfuerzo es curvilínea durante el ejercicio de intensidad progresiva, de esta manera es totalmente proporcional al aumento tanto de la FC como de la intensidad del esfuerzo, logrando de esta manera hallar el UA con mayor facilidad y precisión.

De acuerdo a la capacidad fisiológica de los individuos evaluados encontramos que en la literatura y estudios previos, los valores en porcentaje de acuerdo a la FC encontrada en el UA, es de aproximadamente entre el 80 y el $95 \%$, para deportistas bien entrenados 0 de alto rendimiento (Volkov y col, 1989; Barrios y col, 1995; García y col, 1996; Lozano y cols, 2009, Lozano y cols 2011) observando que en nuestro estudio se encuentra este valor en un $93,47 \pm 2,09$ $\%$, estando dentro de los rangos descritos anteriormente, al momento de determinar la deflexión de la curva de la FC. De igual manera para deportes de conjunto y en especialmente el fútbol de campo, los valores encontrados para estos deportistas en su consumo de oxígeno, oscila entre 50 y $58 \mathrm{ml} . \mathrm{kg}^{-1} \cdot \mathrm{min}^{-1}$ (Wilmore y Costill, 2004; Lozano y cols 2009; Lozano y cols, 2011), observando un valor medio del grupo evaluado dentro de este rango, que fue de $54,23 \pm 3,72$ ml.kg- ${ }^{-1} \cdot \mathrm{min}^{-1}$, teniendo en cuenta la etapa de su preparación ya que se encontraban finalizando año, lo cual encontramos valores bastante altos de acuerdo a otros estudios y en otras etapas de preparación, teniendo en cuenta tanto frecuencia cardiaca máxima, en umbral anaeróbico y volumen máximo de oxígeno.

De igual manera en el estudio realizado por Garcia y cols (2003), se determinó el umbral anaeróbico de una forma matemática $y$ manual, de acuerdo a la cinética de la frecuencia cardiaca, estableciendo un alto grado de confiabilidad por encontrar una muy buena correlación y determinado a jugadores de fútbol de diferentes categorías, de esta manera, asumimos que en el presente estudio la manera de determinar el UA de una forma manual, es muy válida, donde se han encontrado valores en la FC en el momento de determinar el UA en $178,7 \pm 2,09$ ppm y al comparar estos resultados con el estudio llevado a cabo por Garcia y cols (2003), su valor ha sido de $176 \pm 7$ ppm, con un $92,6 \%$ en este parámetro, para ese estudio, con los jugadores profesionales de la Selección de Costa Rica, son muy similares los resultados, tanto en la FC como en su porcentaje, dándole un buen componente de confiabilidad a nuestros resultados.

\section{Conclusiones}

El protocolo interválico de Probst programado en el software TIVREF-v2.0 permite realizar un test de campo específico que se muestra sensible al grado de profesionalización y práctica de los futbolistas, con propósitos claros como es optimizar el rendimiento deportivo, a través de las variables de la frecuencia cardiaca e intensidad de carrera.

Las características de la muestra estudiada permitirá establecer datos de referencia para las categorías juvenil, amateur, semiprofesional y profesional del fútbol Costarricense y de esta manera lograr optimizar el rendimiento de futuras generaciones.

El análisis de la frecuencia cardíaca durante el test ha permitido, en más de un $93 \%$ de los casos, identificar un punto de inflexión que pudiera corresponderse con el umbral anaeróbico, siendo una manera sencilla, confiable y de fácil detección y utilización para tener un control de los deportistas. 
Se han encontrado unos valores en el umbral anaeróbico teniendo en cuenta su frecuencia cardiaca, frecuencia cardiaca máxima, velocidad de carrera y distancia de recorrido, bastante elevados, en relación a estudios anteriores, y es de resaltar que los deportistas evaluados pertenecen a una selección que representará a su país en un evento importante a nivel internacional, como lo será la Copa del Mundo, Brasil 2014.

\section{Agradecimientos}

Nuestro más sincero agradecimiento al profesor Jorge Luis Pinto, por habernos tenido en cuenta, en estas valoraciones con la Selección de Fútbol de Costa Rica, durante su proceso de preparación para la Copa del Mundo, Brasil 2014; de igual manera a la Federación de Fútbol de este país, por brindarnos su apoyo y esfuerzo para estar presentes en sus instalaciones y desarrollar este seguimiento a los deportistas.

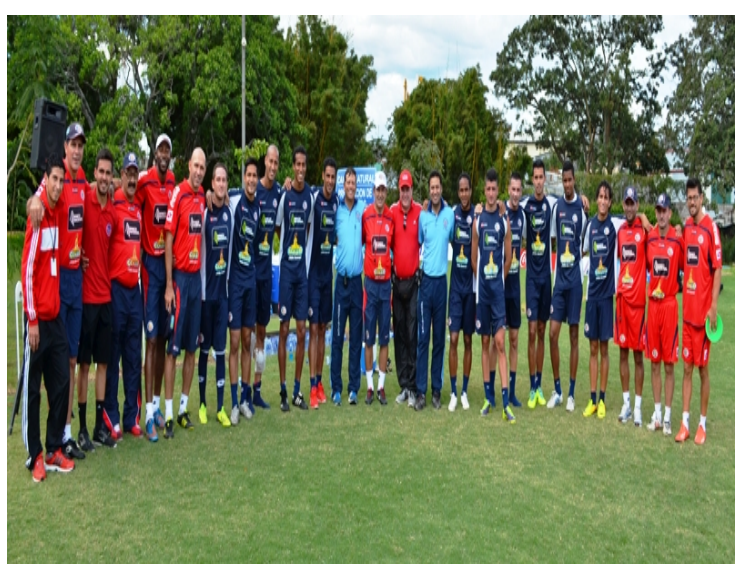

\section{REFERENCIAS BIBLIOGRAFICAS}

American Collage of Sports Medicine. 1999. Manual ACSM, para la Valoración y Prescripción del Ejercicio. Primera Edición. EEUU, Editorial Paidotribo.

Bangsbo, J. 1996. "Yo-yo tests of practical en durance and recovery for soccer". Performance conditioning for soccer 9 (2), p. 8.
Bangsbo, J. 1998. Quantification of anaerobic energy production during intense exercise. Med. Sci. Sports Exerc. 30 (1): 4752.

Barrios, Joaquín R. y Ranzola, A. R. 1995. Manual para el Deporte de Iniciación y Desarrollo. Instituto Nacional de Deportes de Venezuela. pp. 26-37.

Bosco, C.: 1991. Aspectos fisiológicos de la preparación física del futbolista, Barcelona: Ed. Paidotribo.

Conconi, F.; Ferrari, M., Ziglio, P. G.; Droghetti, P.; Codega, L. 1982. Determnation of the anaerobic thereshold by a noninvasive field test in runners. J. Appl. Physiol.. 52(4): 869-873.

Conconi, F. et al. 1996. The Conconi test: Methodology alter 12 years of application. Int. J. Sports Med.. 17: 509 - 519.

Franco, L.: 1998. "Fisiología del baloncesto", Archivos de Medicina del Deporte, 15 (68), pp.471-477.

García Manso. J., Manuel Navarro Valdivieso, José A. Ruiz Caballero. 1996. Bases teóricas del entrenamiento deportivo. Principios y aplicaciones. Editorial Gymnos. Madrid.

García, L. J.; Villa V. J.; Rodríguez M., A; Morante, J. C.; Alvarez, E. y Jover, R. 2003. Aplicación de un test de esfuerzo interválico (Test de Probst) para valorar la cualidad aeróbica en futbolistas de la liga española. Apunts, Educación Física y Deportes. 71, pp. 80-88.

Hofmann, P.; Pokan, R.; Preidler, K.; Leitner, H.; Szolar, D.; Eber, B. y Scwaberger, G.: 1994. "Relationship between heart rate threshold, lactate turn point and myocardial function", Int. J. Sports Med, 15 (5), pp. 232-237. 
Hofmann, P.; Pokan, R.; Von Duvillard, S. P.; Seibert, F. J.; Zweiker, R.; Schmid, P.: 1997. "Heart rate performance curve during incremental cycle ergometer exercise in healthy young male subjects", Med, Sci. Sports Exerc., 29 (6), pp. 762-768.

López C., J, y Legido, J. 1991. Umbral Anaeróbico. Bases Fisiológicas y Aplicación. Ed. Interamericana. McGraw-Hill. Madrid..

López Chicharro J., Aznar L. Susana, Vaquero Almudena F., López M. Luis M., Mulas Alejandro L., y Ruiz Margarita Pérez. 2004. Transición Aeróbica - Anaeróbica, Concepto, metodología de determinación y aplicaciones. 1ra Ed. Enero,. Edición Master Line \& Prodigio S. L.

Lozano Z., R. Enrique. 2009. Determinación del Umbral Anaeróbico en patinadores de velocidad sobre ruedas a través de un test de campo. Revista Actividad Física y Desarrollo Humano, Facultad de Salud. Universidad de Pamplona. Volumen 1, enero a diciembre. Páginas 66 - 76.

Lozano, R. Enrique; Mariño, Nelson y Clavijo Nelson. 2011. Determinación del umbral anaeróbico en jugadores profesionales de fútbol, a través de la frecuencia cardiaca. EFDeportes.com, Revista Digital. Buenos Aires, Año 16, No 163, Diciembre. http://www.efdeportes.com. Probst, H.; Comminot, C.H. y Rojas, J. 1989. Conconi-test auf dem Fahrradergometer. Schwiez Z Sportmed,. $\mathrm{n}$. 37 , pp. 141-147.

Reilly, T. 1997. Energetics of high-intensity exercise (soccer) with particular reference to fatigue. J. Sport Sci. 15: 257-263.

Villa, J.G.; García-López, J.; Morante, J.C. y San Roman, Z. 2000. Validación del test de resistencia específico de Probst en futbolistas profesionales. Archivos de Medicina del Deporte. 17(76): 185-186.

Villa, J. G.; Rodríguez-Marroyo, J. A.; Vaquera, A.; Morante, J. C.; García-López, J.; Ávila, C.; Seco J.; Pérez, J. A. y Cejuela,
R. 2007. Mejoras en esfuerzos y recuperación tras entrenamiento de pretemporada mediante test de campo TIVRE-Basket. En S. J. Ibáñez y M. M. Macías (Eds.). IV Congreso Ibérico de Baloncesto. Cáceres.

Volkov, V.M. y Filin, V.P. 1989. Selección Deportiva. Moscú. Ed. Ráduga.

Wilmore, Jack H. and Costill, David L. 2004. Fisiología del Esfuerzo. $5^{\text {a }}$ Edición. Editorial Paidotribo. Barcelona. 